\title{
The genomes and mechanisms of adaptation to the cold climates in Russian native cattle breeds
}

\author{
Laura Buggiotti \\ Royal Veterinary College, University \\ of London, London, UK \\ Institute of Cytology and Genetics, \\ Novosibirsk, Russia
}

\author{
Andrey Yurchenko \\ Kurchatov Genomics Center, \\ Institute of Cytology and Genetics, \\ Novosibirsk, Russia \\ Nikolay Yudin \\ Kurchatov Genomics Center, \\ Institute of Cytology and Genetics,
}

\author{
Denis M. Larkin \\ Royal Veterinary College, University \\ of London, London, UK \\ Institute of Cytology and Genetics, \\ Novosibirsk, Russia
}

\section{Keywords - adaptation, cattle, missense mutation}

The genetic structure, history and signatures of selection were revealed using the sequenced genomes of two native Russian cattle breeds: the Yakut cattle and the Kholmogory. Twenty individuals from each breed have been resequenced to $\sim 10-11 \mathrm{x}$ filtered coverage and combined with the genome sequences of two most closely related cattle breeds: Hanwoo and Holsten. Our combined dataset contained over 31 million high-quality SNPs. This set has been used to detect copy number variants (CNVs) in the four breed genomes. The CNVs found in the Yakut and breed were enriched in genes from the gene ontology terms regulation of actin filament-based processes, MHC protein complex, and antigen processing and presentation could potentially contribute to adaptation to local environments. There were 299 CNVRs shared between the Yakut and Kholmogory breeds, of which the ACTG1 (actin gamma 1) was the most frequent CNVR and covered $>90 \%$ of the gene. Our SMC analysis had demonstrated a different $N_{\mathrm{e}}$ trajectory for the Yakut cattle compared to the other three breeds and additional Chinese and Indian indicine cattle breeds, while the Kholmogory's one was close to Holstein. The demographic history of the Yakut cattle was further analyzed with the TreeMix, $f 3$ and D4 statistics and the RFMix. The TreeMix results indicated no admixture between the Yakut cattle and other cattle breeds and related bovinae species. While the RFMix data suggested introgression of indicine cattle in to the Yakut cattle genomes. We further looked into the Yakut genome regions likely being introduced from other cattle populations. We found that the corresponding SNP alleles often are present in other bovinae genomes, with very few of them were indicine-cattle specific. This suggests that the Yakut cattle could have genome regions containing ancestral alleles, removed from the European taurine genomes. We looked into the high-frequency missense mutation found in the Yakut cattle. Out of 48 such mutations 21 were found in the SPTBN5 gene. Spectrin 5 is an actin-binding gene involved in resistance to parasites in humans, suggesting that it could be related to parasite resistance in the Yakut cattle as well. Spectrin 5 region had the highest $\mathrm{F}_{S T}$ between the Yakut cattle and over 3000 cattle individuals from the 1000 bull genomes project. The only exclusive Yakut-specific missense change absent from any other cattle breeds and other bovinae species was found in the NRAP gene. Interestingly, the same amino acid change observed in the Yakut cattle was observed in the vast majority of mammals that enter either torpor or hibernation suggesting that this gene, expressed exclusively in the heart and skeletal muscles could be related to mechanisms of slowing metabolism in the Yakut cattle during the cold winter months. This an important step toward revealing the history, and signatures adaptation within the native Russian cattle made to facilitate application of genomic technologies to improve breeding procedures and to increase the effectiveness of genomic selection for livestock in Russia.

\section{Acknowledgment}

This study was funded in part by the RFBR grant 18-016-00185 and the RSF grant 19-76-20026. 